High Beck Depression Inventory 21 scores in adolescents without depression are associated with negative self-image and immature defense style

Savilahti, Emma M.

2018-05

Savilahti , E M , Haravuori , H , Rytilä-Manninen , M , Lindberg , N , Kettunen , K \&

Marttunen, M 2018 , ' High Beck Depression Inventory 21 scores in adolescents without depression are associated with negative self-image and immature defense style ' , Psychiatry Research, vol. 263 , pp. 61-68 . https://doi.org/10.1016/j.psychres.2018.02.040

http://hdl.handle.net/10138/302399

https://doi.org/10.1016/j.psychres.2018.02.040

publishedVersion

Downloaded from Helda, University of Helsinki institutional repository.

This is an electronic reprint of the original article.

This reprint may differ from the original in pagination and typographic detail.

Please cite the original version. 


\title{
High Beck Depression Inventory 21 scores in adolescents without depression are associated with negative self-image and immature defense style
}

\author{
Emma M. Savilahti ${ }^{\mathrm{a}, *, 1}$, Henna Haravuori ${ }^{\mathrm{a}, \mathrm{b}}$, Minna Rytilä-Manninen ${ }^{\mathrm{a}, \mathrm{c}, 2}$, Nina Lindberg ${ }^{\mathrm{d}, 3}$, \\ Kirsi Kettunen ${ }^{\mathrm{a}, \mathrm{c}, 2}$, Mauri Marttunen ${ }^{\mathrm{a}, \mathrm{b}}$ \\ a Adolescent Psychiatry, University of Helsinki and Helsinki University Hospital, PO BOX 660, 00029 HUS Helsinki, Finland \\ ${ }^{\mathrm{b}}$ Mental Health Unit, National Institute for Health and Welfare, Helsinki, Finland \\ ${ }^{\mathrm{c}}$ Hospital District of Helsinki and Uusimaa, Kellokoski Hospital, Kellokoski, Finland \\ ${ }^{\mathrm{d}}$ Forensic Psychiatry, University of Helsinki and Helsinki University Hospital, Helsinki, Finland
}

\section{A R T I C L E I N F O}

\section{Keywords:}

Adolescence

Adverse life events

Defense Style Questionnaire (DSQ)

Offer Self-Image Questionnaire (OSIQ)

Inpatient

Psychiatric self-report

Subthreshold depression

\begin{abstract}
A B S T R A C T
Beck Depression Inventory (BDI) is widely used in assessing adolescents' psychological wellbeing, but occasionally the result diverges from diagnostics. Our aim was to identify factors associated with discrepancies between BDI scores and diagnostic assessment in adolescent psychiatric patients and general population.

The study comprised 206 inpatients (13-17 years old) and 203 age and gender matched non-referred adolescents. Study subjects filled self-reports on depression symptoms (BDI-21), alcohol use (AUDIT), defense styles (DSQ-40) and self-image (OSIQ-R), and on background information and adverse life events. Diagnostics was based on K-SADS-PL interview, and/or clinical interview and clinical records when available.

We compared subjects who scored in BDI-21 either 0-15 points or 16-63 points firstly among subjects without current unipolar depression $(n=284)$, secondly among those with unipolar depression $(n=105)$. High BDI-21 scores in subjects without depression diagnosis $(n=48)$ were associated with female sex, adverse life events, parents' psychiatric problems, higher comorbidity, higher AUDIT scores, worse self-image and more immature defense styles. Low BDI-21 scores among subjects with depression diagnosis $(\mathrm{n}=23)$ were associated with male sex, more positive self-image and less immature defense style.

In conclusion, high BDI-21 scores in the absence of depression may reflect a broad range of challenges in an adolescent's psychological development.
\end{abstract}

\section{Introduction}

Major depressive disorder (MDD) is one of the most common psychiatric disorders in adolescence with a cumulative prevalence of up to $20 \%$ (Avenevoli et al., 2015). It often leads to a decrease in cognitive and social functioning and increases the risk for suicidality. Furthermore, depressive symptoms that do not reach the diagnostic threshold of MDD (prevalence 5-29\%) also cause significant impairment (Carrellas et al., 2017). Several screening and diagnostic tools for depression have therefore been developed (Brooks and Kutcher, 2001; Stockings et al., 2015). In clinical practice, self-reported depressive symptoms and clinical diagnostics occasionally diverge raising the question what could explain this discrepancy. To our knowledge, this issue has not been studied in adolescents.

Research data on the risk factors for depression point to factors worth considering also in subthreshold depression. The three most important risk factors for depression in adolescents are female sex, a family history of depression and exposure to psychosocial stress (Thapar et al., 2012). The intergenerational transmission of depressive symptoms arises from a mix of hereditary and environmental factors (Mason et al., 2017; Weissman et al., 2006). Various psychosocial stress factors can induce depression in adolescents (St Clair et al., 2015; Rice et al., 2017), and susceptibility appears to be higher in females than males (St Clair et al., 2015). Depressive symptoms in adolescents are also associated with psychological factors, in particular negative selfimage (Fine et al., 1993; Erkolahti et al., 2003) and immature styles

\footnotetext{
* Corresponding author.

E-mail addresses: emma.savilahti@hus.fi (E.M. Savilahti), henna.haravuori@hus.fi (H. Haravuori), minna.rytila-manninen@hus.fi (M. Rytilä-Manninen), nina.lindberg@hus.fi (N. Lindberg), kirsi.kettunen@fimnet.fi (K. Kettunen), mauri.marttunen@hus.fi (M. Marttunen).

${ }^{1}$ Address: Adolescent Psychiatry, Helsinki University Hospital, PO BOX 660, 00029 HUS, Helsinki, Finland.

2 Address: Kellokoski Hospital, Ohkolantie 20, 04500 Kellokoski, Finland.

${ }^{3}$ Address: Psykiatriakeskus, P O Box 590, 00029 HUS, Helsinki.
} 
(Chan, 1997; Muris et al., 2003; Ruuttu et al., 2006).

For identifying depressive symptoms, one of the most widely used structured self-reports is Beck Depression Inventory (BDI) - 21 (Beck et al., 1961). This 21-item depression scale has been validated for adolescents (Stockings et al., 2015). BDI-21 does not, however, directly screen the DSM depression criteria and stresses cognitive symptoms. For diagnostics in adolescents the gold standard is the semi-structured clinical interview called The Schedule for Affective Disorders and Schizophrenia for School-Age Children-Present and Lifetime version (KSADS-PL) (Kaufman et al., 1997; Ambrosini, 2000). Studies that compare the results of BDI and clinical diagnostics usually aim at defining the psychometrics of BDI (Kumar et al., 2002; Osman et al., 2008; Dolle et al., 2012). Our aim, in contrast, is to investigate what psychological and background factors explain why the self-report and the diagnostic appraisal may diverge. We compare the BDI-21 scores and psychiatric diagnostics among both psychiatric inpatients and control subjects from general population. Our premise is to consider the clinicians' assessments as the gold standard for psychiatric diagnostics, while acknowledging that the diagnostics in adolescent psychiatry entails uncertainties (Lauth et al., 2010; Youngstrom et al., 2011). Drawing on research on the risk factors for depression, our hypothesis is that divergence between the absence of unipolar depression diagnosis and high BDI-21 scores is associated with immature defense styles and negative self-image.

\section{Methods}

\subsection{Participants}

\subsubsection{Patients}

The participants and clinical procedures have been described previously (Rytila-Manninen et al., 2014). The Kellokoski Hospital Adolescent Inpatient Follow-Up Study (KAIFUS) is a longitudinal, naturalistic study on clinical characteristics, psychometrics and the impact of treatment in adolescents (13-17 years old) who were hospitalized in adolescent psychiatry for the first time in their life between September 2006 and August 2010 ( $\mathrm{n}=395$ ). All participants and their legal guardians received verbal and written information about the study and gave thereafter their written informed consent. The Ethics Committee of the Helsinki University Hospital approved the study protocol, and the institutional authority at the Hyvinkää Hospital Area granted permission to conduct the study. Study participation required sufficient knowledge of the Finnish language and adequate cognitive capacity, as well as a hospital treatment period of at least two weeks. Of 395 adolescent patients, 315 were eligible. In $62(16.4 \%)$ cases, the adolescent or his/her parents/guardians did consent to participation. In 23 cases (6\%), patients or their parents/guardians discontinued treatment, and 24 cases (6\%) provided incomplete data. The final sample consisted of 206 adolescents: 60 (29.1\%) boys and $146(70.9 \%)$ girls. Study nonparticipation was not related to age $(\mathrm{p}=0.31)$, socio-economic status (SES, $\mathrm{p}=0.38)$, living situation $(\mathrm{p}=0.58)$, or having a primary diagnosis of substance use $(\mathrm{p}=0.59)$, $\operatorname{mood}(\mathrm{p}=0.92)$, anxiety ( $\mathrm{p}=0.39)$, eating $(p=0.34)$, or conduct disorders $(p=0.09)$. It was, however, associated with male gender $(p=0.02)$ and psychotic disorders ( $p=0.02$ ) (Rytila-Manninen et al., 2014).

\subsubsection{Community sample}

The comparison group was recruited from the same geographical area as the patient group. It consisted of a random sample of sex- and age-matched students from seven schools (two high/secondary schools, one vocational school and four middle/comprehensive schools). Of the 474 invited students, $43.0 \%(n=203)$ completed the interview and the questionnaires, $42.5 \%(\mathrm{n}=202)$ refused to participate, and $14.5 \%$ (n $=68$ ) did not complete the questionnaires despite providing consent. The final comparison group consisted of 203 adolescents. There were no significant differences between completers and non-completers in regards to socioeconomic status $(\mathrm{p}=0.61)$ or living situation $(\mathrm{p}=0.49)$. For adolescents who completed the K-SADS-PL-interview, a treatment referral was endorsed when appropriate.

\subsection{Diagnostics and psychometrics}

\subsubsection{Psychiatric diagnostics}

Medical doctors who specialized in adolescent psychiatry evaluated the psychiatric diagnostics according to DSM-IV and based on clinical records, which were available for patients, and K-SADS-PL which was conducted by experienced psychiatric nurses trained in K-SADS-PL. The K-SADS-PL is a semi-structured diagnostic interview that has good to excellent test-retest reliability and high concurrent validity and interrater agreement for the original and translated versions (Kaufman et al., 1997; Ambrosini, 2000). The Finnish translation has been used in studies of adolescent inpatient and outpatient settings (Tuisku et al., 2006; Mustanoja et al., 2011). If a patient did not cooperate sufficiently for conducting K-SADS-PL reliably, psychiatric diagnostics was based on clinical interview, observation in the hospital and clinical records. Diagnostic meetings were held during data collection, and any discrepancies were settled by consensus between three experienced adolescent psychiatrists (H.H, N.L, K.K).

\subsubsection{Socio-demographic factors and adverse life events}

Study subjects were interviewed on socio-demographic factors as well as adverse life events and stressors with a structured questionnaire composed for this study and as part of the K-SADS-interview as described in a previous publication (Rytila-Manninen et al., 2014). Their answers to questions on adverse life events and stressors were categorized as yes or no. In the K-SADS-PL interview, school bullying was screened in the school adaptation and social relationship section. In the post-traumatic stress disorder screening section of K-SADS-PL, domestic violence, exposure to physical and/or sexual abuse was inquired. In the structured background data questionnaire SES was assessed by asking "What is your father's occupation?", or if an adolescent lived with his/ her mother (and stepfather), we recorded mother's occupation. SES was classified as high when the guardian (primarily the father) was a selfemployed worker or upper-level employee, middle when the guardian was a lower-level employee or manual worker, and low if the guardian was retired, a student or unemployed. Subjects were also asked about parental divorce and whether he/she knew if his/her mother or father suffered from psychiatric or substance use problems requiring professional help. One question from the Life Events Checklist was used to record parents' criminality (Has your parent ever been arrested, suspected or judged for a legal offense?). In the patient group, clinical records additionally provided information on the family background as supplied by legal guardian(s)/parent(s).

\subsubsection{Self-reports on psychiatric symptoms and psychological factors}

Study participants, both patients and control subjects, filled in structured self-reports on psychiatric symptoms and psychological factors.

BDI-21 is a 21 -item self-report scale of depressive symptoms that has been validated for adolescents (Stockings et al., 2015).

Alcohol Use Disorders Identification Test (AUDIT) is a self-report scale screening for alcohol misuse, and in the extended version used in this study, also enquires about other substance use. It has been shown to be applicable to adolescents (Knight et al., 2003).

Defense Style Questionnaire (DSQ) - 40 a reliable and valid selfreport instrument for adolescents. In adolescents, it appears to discriminate better four defense styles (mature, neurotic, image-distorting, and immature) rather than three, which is alternatively used in adult populations (Ruuttu et al., 2006).

Offer Self-Image Questionnaire (OSIQ) is a self-report inventory containing descriptive statements with six-point Likert-type scale. The OSIQ has been widely used to assess the self-image of adolescents, and 
it has been validated among Finnish adolescents (Laukkanen et al., 2009). In the current study, we used the revised version OSIQ-R. It consists of 129 items, which make up 12 component scales: 1) emotional tone (ET) measures the affective harmony and stability, 2) impulse control (IC) measures the strength of the ego in handling pressure without resorting to inappropriate action, 3) mental health ( $\mathrm{MH})$ measures the presence or absence of psychopathological thoughts, 4) social functioning (SF) 5) family functioning (FF), 6) Self-Confidence (SC, former mastery of the External World), 7) Self-reliance (SR, former Superior Adjustment), measures how well the adolescent copes with her/himself and the world around, 8) Ethical Values (EV, former morals), 9) body image (BI), 10) Vocational attitude (VA) measures how well the adolescent is faring in accomplishing the tasks of learning and planning his/her future, 11) Sexuality (SX), 12) Idealism. In the current study, we focused on the scales that are most relevant in relation to depressive symptoms and thus omitted sexuality, vocational attitude and idealism. Furthermore, the sexuality scale reportedly has a U-curve nature and poor reliability. Also the idealism scale has poorer reliability than other scales in OSIQ and moreover, it reflects more cognition than emotions (Offer et al., 1992). We used the raw scores because the OSIQ-R has not been normalized in European populations. In raw scores, the higher the score, the worse the self-image.

\subsection{Data analysis}

The key question was how the self-reports of study subjects differed from clinical diagnostic appraisal. Thus, we examined what factors differentiated those who scored low on BDI-21 from those who scored high among subjects without a diagnosis of current unipolar depression, and among subjects with a diagnosis of current unipolar depression. We performed the analyses in the whole population as well as separately in the patient population and the control population.

We ran Receiver Operating Characteristic (ROC) analyses for BDI-21 with current depression diagnosis.

We chose the cutoff point for BDI based on the ROC analyses (ref. 3.1 Distributions, ROC analyses and correlations) and previous publications. In depression studies on adolescents, a sum score of 16 has been most widely used as a BDI-21 cut-off point for identifying MDD (Stockings et al., 2015). Based on the chosen cutoff point, we created a dichotomous variable of BDI-21 scores.

We compared with Pearson's chi square $(\chi 2)$ test in subjects not meeting the DSM-IV criteria for current unipolar depression the frequencies of background characteristics (sex, SES, parent's psychiatric care and substance use), adverse life events (parents' divorce, parent's death, exposure to physical abuse at home, exposure to physical abuse outside of home, witnessing intimate partner violence, sexual abuse victimization, being bullied at school), DMS-IV diagnostic groups between subjects grouped according to the BDI-21 cutoff point (16 points). Since not all variables were normally distributed, we used Mann Whitney $U$-test to compare in afore mentioned groups the distribution of age, scales of self-image (measured with OSIQ-R) and scales of defense styles (measured with DSQ-40 questionnaire) and AUDIT sum.

Secondly, we ran the afore described analyses for subjects who met the DSM-IV criteria for current unipolar depression and compared likewise subjects grouped according to the BDI-21 cutoff point.

We checked the correlations between BDI-21 scores and OSIQ-R areas and DSQ-40 defense styles with Spearman correlation.

We performed among subjects without depression binomial logistic regression analysis with the dichotomous BDI-21 variable (under 16 points or 16 points and over) as dependent variable and as independent variables study group (patient or control), sex, the number of psychiatric diagnoses, parent's psychiatric problems, defense style scores (DSQ-40) as well as the OSIQ-R component scores.
Table 1

Characteristics of the study population: comparison between patients and control subjects.

\begin{tabular}{|c|c|c|c|}
\hline & $\begin{array}{l}\text { PATIENTS } \\
206 \\
\text { n }(\%)\end{array}$ & $\begin{array}{l}\text { CONTROLS } \\
203 \\
\text { n }(\%)\end{array}$ & $\mathrm{p}\left(\mathrm{X}^{2}\right)$ \\
\hline male & 60 (29) & $55(27)$ & 0.6 \\
\hline age median (min-max) & $15(13-17)$ & $15(13-17)$ & 0.1 (MWø) \\
\hline SES*: high & $19(9)$ & 30 (15) & 0.000 \\
\hline SES*: middle & $78(38)$ & $109(54)$ & \\
\hline SES*: low & 109 (53) & $64(31)$ & \\
\hline previous psychiatric care & $190(92)$ & $16(8)$ & 0.000 \\
\hline $\begin{array}{l}\text { any current psychiatric DSM-IV } \\
\text { diagnosis }\end{array}$ & $206(100)$ & $44(22)$ & 0.000 \\
\hline $\begin{array}{l}>1 \text { current psychiatric DSM-IV } \\
\text { diagnosis }\end{array}$ & $141(68)$ & $8(4)$ & 0.000 \\
\hline $\begin{array}{l}\text { subjects with any current DSM-IV } \\
\text { unipolar depression diagnose }\end{array}$ & 117 (57) & $3(1)$ & 0.000 \\
\hline Major depressive disorder & $102(49)$ & $1(0.5)$ & \\
\hline Depressive disorder NOS & $14(7)$ & $1(0.5)$ & \\
\hline Dysthymic Disorder & $2(1)$ & $1(0.5)$ & \\
\hline $\begin{array}{l}\mathrm{BDI}-21 \dagger \\
\text { median (min-max; n) }\end{array}$ & $22(0-55 ; 186)$ & $1(0-31 ; 203)$ & $\begin{array}{l}0.000 \\
(\mathrm{MW} \mathbf{m})\end{array}$ \\
\hline $\begin{array}{l}\text { AUDIT }+ \\
\text { median (min-max; } n)\end{array}$ & $0(0-31 ; 192)$ & $1(0-28 ; 201)$ & 0.6 (MWa) \\
\hline
\end{tabular}

* socio-economic status, $\dagger$ BDI Beck depression inventory.

$\ddagger$ AUDIT Alcohol Use Disorders Identification Test, =MW Mann-Whitney $U$-test for independent samples.

\section{Results}

\subsection{Distributions, ROC analyses and correlations}

Current unipolar depression DSM-IV diagnoses in the entire study population were distributed as follows: 1) the most common diagnosis was major depressive disorder (MDD, 296.20-296.35) as first diagnosis in 92, second diagnosis in 9 and third diagnosis in 2 subjects, 2) Depressive disorder NOS (311) as first diagnosis in 8, second diagnosis in 6 subjects and third diagnosis in 1 subject, 3) Dysthymic Disorder (300.4) as first diagnosis in 1 and as second diagnosis in 1 subject (Table 1). Overview and comparison of the distribution of background factors, diagnostics and psychometrics in the patient and control populations are depicted in Table 1. Patients had more psychiatric morbidity and their socio-economic status was lower compared with control subjects (Table 1). They scored higher in BDI-21, but not in AUDIT (Table 1) compared with control subjects.

The distributions of BDI-21 in the patient (Fig. 1A) and control (Fig. 1B) populations differed starkly. ROC curve for BDI-21 in this study population showed rather good accuracy for current depression diagnosis: The area under curve was 0.87 (95\% confidence interval 0.84-0.91). For the optimal cutoff point, the point closest to $(0,1)$ point was at BDI-21 point 15.5, whereas the Youden index suggested the optimal cutoff point to be 9.5. Assessing these results together with previous studies (Stockings et al., 2015), we chose the cutoff point of 16.

BDI-21 scores were positively correlated with all scales $(1-9)$ of OSIQ-R used in this study, i.e. more severe depression symptoms in BDI21 correlated with worse self-image (Table S1 in Supplement). BDI-21 scores were positively correlated with immature defense style measured with DSQ-40, and negatively correlated with mature defense style (Table S1 in online supplement).

\subsection{Subjects without unipolar depression: comparisons based on BDI-21 dichotomous variable}

Forty eight subjects scored high in BDI-21 (sum score 16 or above) even when they did not meet the DSM-IV criteria for current unipolar depression; 36 in the patient group and 12 in the control group. For the 
A)

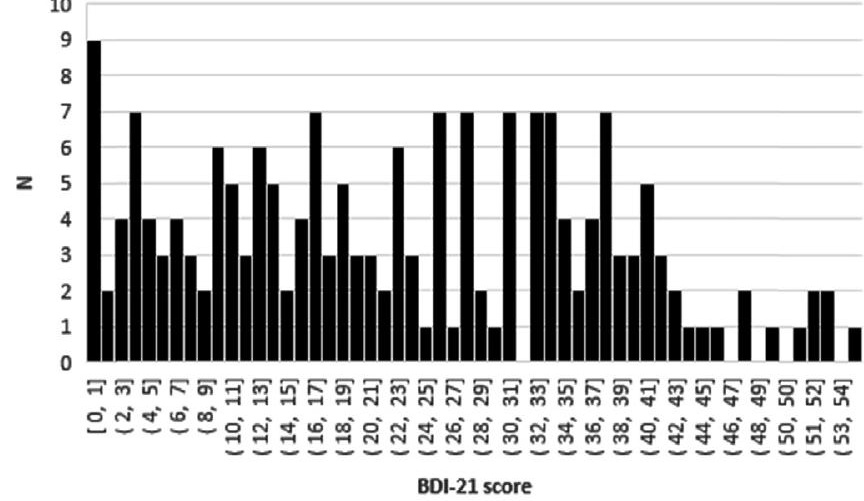

B)

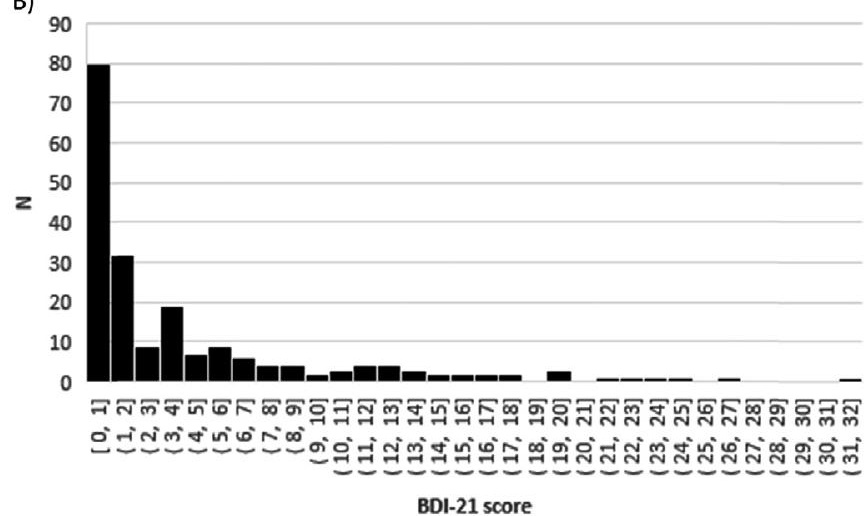

Fig. 1. Distribution of Beck Depression Inventory (BDI) - 21 scores among A) patients and B) control subjects. N.B. The axes are in different scales in A and B.

analyses on background variables, diagnoses, OSIQ-R and DSQ, shown are only results (Tables $2-5)$ with statistical significance $(\mathrm{p}<0.05)$ in at least one of the groups (whole population, patients only, control subjects only).

\subsubsection{Sex, age, adverse life events and parental psychosocial problems}

Subjects who scored high in BDI-21 even when they did not have current unipolar depression were more often female and in late adolescence (Table 2). They reported more often adverse life events and their parents having more often psychiatric care and/or treatment due to substance use problems than those scoring low in BDI-21 (Table 2). These differences were seen mainly in the control population where the overall prevalence of adverse life events and social problems was low, in contrast to the patient population (Table 2).

\subsubsection{Psychiatric diagnoses}

Only 2 of subjects scoring high in BDI-21 without current depression had no psychiatric diagnosis (Table 3) and these subjects belonged to the control population. Bipolar and other mood disorders, anxiety disorders, eating disorders and substance use disorder were more prevalent in these subjects than in those scoring low in BDI and no depression (Table 3). The differences were not as significant when the populations of patients and control subjects were examined separately (Table 3).

\subsubsection{Self-image and psychological defense mechanisms}

The self-image (measured with 9 scales of OSIQ-R) of subjects scoring high in BDI-21 but no depression was worse compared with subjects scoring low in BDI-21 and no depression (Table 4). In the control population, the difference was seen in all nine scales of OSIQ-R, whereas within the patient population the differences in the scales of morals and self-confidence were not statistically significant (Table 4).

Psychological defense mechanisms were measured with DSQ-40. BDI-21 scores 16 and higher in subjects without depression diagnosis were associated with less manifestation of mature defenses and more of immature defenses compared with subjects scoring low in BDI-21 and no depression (Table 4). Moreover, in the patient population, neurotic defenses were more common in subjects scoring high in BDI-21 and no depression (Table 4). In the control population, image distorting defenses were associated with high BDI-21 scores without depression compared with low BDI-21 scores and no depression.

\subsubsection{AUDIT}

In all subjects without depression diagnosis, higher AUDIT scores were associated with BDI-21 scores 16 or over compared with lower BDI-21 scores (Table 4).

\subsubsection{Regression analysis}

Binomial logistic regression analysis among subjects without depression was statistically significant, $\chi^{2}(13)=136, p<0.0000$ ( $n=248$, missing 41). The Variance Inflation Factor (VIF) of the variables varied between 1.3 and 6.5. The model explained $73 \%$ (Nagelkerke $R^{2}$ ) of the variance in BDI-21 dichotomous group and correctly classified $94 \%$ of cases. It showed, however, no single factor (patient/control,gender, the number of psychiatric diagnoses, parent's psychiatric problems, DSQ-40 scores, OSIQ-R component scores) to greatly contribute to the BDI-21 score group (0-15 points or 16 and

Table 2

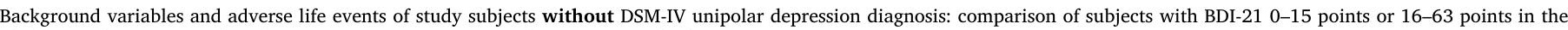
whole population (controls and patients), among patients and among control subjects.

\begin{tabular}{|c|c|c|c|c|c|c|c|c|c|}
\hline & \multicolumn{3}{|l|}{ ALL } & \multicolumn{3}{|l|}{ PATIENTS } & \multicolumn{3}{|l|}{ CONTROLS } \\
\hline & BDI 0-15p & BDI 16-63 p & $\mathrm{p}\left(\mathrm{X}^{2}\right)$ & BDI 0-15p & BDI $16-63 p$ & $\mathrm{p}\left(\mathrm{X}^{2}\right)$ & BDI $0-15 p$ & BDI $16-63 p$ & $\mathrm{p}\left(\mathrm{X}^{2}\right)$ \\
\hline n (\%) & $236(83)$ & $48(17)$ & & $48(57)$ & $36(43)$ & & $188(94)$ & $12(6)$ & \\
\hline male & $80(34)$ & $8(17)$ & 0.019 & $27(56)$ & $6(17)$ & 0.000 & $53(28)$ & $2(17)$ & 0.4 \\
\hline age mean (median; range; Mann-Whitney test) & $15(13-17)$ & $15(13-17)$ & 0.03 & $15(13-17)$ & $15(13-17)$ & 0.05 & $15(13-17 ; 188)$ & $15(14-17 ; 12)$ & 0.40 \\
\hline previous psychiatric care & $51(22)$ & $40(83)$ & 0.000 & $42(88)$ & $35(97)$ & 0.1 & $9(5)$ & $5(42)$ & 0.000 \\
\hline taken into custody & $16(9)$ & $8(17)$ & 0.025 & $14(29)$ & $8(22)$ & 0.5 & $2(1)$ & $0(0)$ & 0.7 \\
\hline parents' divorce & $98(4)$ & $29(60)$ & 0.016 & $31(65)$ & 25 (69) & 0.6 & $67(36)$ & $4(33)$ & 0.9 \\
\hline parents' psychiatric problems & $29(12)$ & $25(52)$ & 0.000 & $13(27)$ & $21(58)$ & 0.004 & $16(9)$ & $4(33)$ & 0.005 \\
\hline parent's substance use problems & $23(10)$ & $18(38)$ & 0.000 & $17(35)$ & $16(47)$ & 0.4 & $6(3)$ & $2(17)$ & 0.02 \\
\hline domestic violence & $29(12)$ & $20(42)$ & 0.000 & $18(38)$ & 17 (47) & 0.4 & $11(6)$ & $3(25)$ & 0.01 \\
\hline physical abuse & $12(5)$ & $13(27)$ & 0.000 & $7(15)$ & $11(31)$ & 0.055 & $5(3)$ & $2(17)$ & 0.01 \\
\hline sexual abuse & $7(3)$ & $12(25)$ & 0.000 & $6(13)$ & $12(33)$ & 0.01 & $1(1)$ & $0(0)$ & 0.8 \\
\hline victim to school bullying & 31 (13) & $15(31)$ & 0.001 & $20(42)$ & $15(42)$ & 0.9 & $11(6)$ & $0(0)$ & 0.4 \\
\hline other trauma & 35 (15) & $18(38)$ & 0.000 & $23(48)$ & $15(42)$ & 0.6 & $12(6)$ & $3(25)$ & 0.02 \\
\hline
\end{tabular}

Statistical significance $\mathrm{p}<0.05$ is marked in bold. 
Table 3

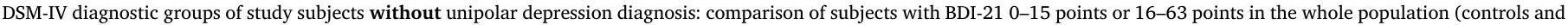
patients), among patients and among control subjects.

\begin{tabular}{|c|c|c|c|c|c|c|c|c|c|}
\hline & \multicolumn{3}{|l|}{ ALL } & \multicolumn{3}{|l|}{ PATIENTS } & \multicolumn{3}{|l|}{ CONTROLS } \\
\hline & BDI $0-15 p$ & BDI $16-63 p$ & $\mathrm{p}\left(\mathrm{X}^{2}\right)$ & BDI $0-15 p$ & BDI $16-63 p$ & $\mathrm{p}\left(\mathrm{X}^{2}\right)$ & BDI $0-15 p$ & BDI $16-63 p$ & $\mathrm{p}\left(\mathrm{X}^{2}\right)$ \\
\hline n (\%) & $236(83)$ & $48(17)$ & & $48(57)$ & $36(43)$ & & $188(94)$ & $12(6)$ & \\
\hline no psychiatric diagnosis & $158(67)$ & $2(4)$ & 0.000 & 0 & 0 & & $158(84)$ & $2(17)$ & 0.000 \\
\hline 1 psychiatric diagnosis & 45 (19) & $16(33)$ & & $18(38)$ & $9(25)$ & 0.3 & $27(14)$ & $7(58)$ & \\
\hline 2 psychiatric diagnoses & $23(10)$ & $24(50)$ & & $20(42)$ & $21(58)$ & & $3(2)$ & $3(25)$ & \\
\hline 3 psychiatric diagnoses & $10(10)$ & $6(13)$ & & $10(21)$ & $6(17)$ & & 0 & 0 & \\
\hline bipolar/other mood do ${ }^{a}$ & $4(2)$ & $10(21)$ & 0.000 & $4(8)$ & $10(28)$ & 0.02 & 0 & 0 & 1 \\
\hline conduct do & $16(7)$ & 9 (19) & 0.008 & 14 (29) & $8(22)$ & 0.5 & $2(1)$ & $1(8)$ & 0.045 \\
\hline anxiety do & $21(9)$ & $18(38)$ & 0.000 & $12(25)$ & 14 (39) & 0.2 & $9(5)$ & $4(33)$ & 0.000 \\
\hline substance abuse & $16(7)$ & $9(19)$ & 0.04 & $6(13)$ & $5(14)$ & 0.7 & $10(5)$ & $4(33)$ & 0.000 \\
\hline eating do & $8(3)$ & $5(10)$ & 0.03 & $4(8)$ & $5(14)$ & 0.4 & $4(2)$ & 0 & 0.6 \\
\hline psychosis & $13(6)$ & $5(10)$ & 0.2 & $12(25)$ & $5(14)$ & 0.2 & $1(0.5)$ & 0 & 0.8 \\
\hline OCD & $1(0.4)$ & $3(6)$ & 0.02 & $1(2)$ & $3(8)$ & 0.2 & 0 & 0 & 1 \\
\hline $\mathrm{ADHD} / \mathrm{ADD}$ & $14(6)$ & $2(4)$ & 0.6 & $10(21)$ & $2(6)$ & 0.05 & $4(2)$ & 0 & 0.6 \\
\hline autism & $7(3)$ & $2(4)$ & 0.7 & 7 (15) & $2(7)$ & 0.2 & 0 & 0 & 1 \\
\hline other $\mathrm{dg}$ & $7(3)$ & $8(17)$ & 0.000 & $7(15)$ & $8(22)$ & 0.3 & 0 & 0 & 1 \\
\hline
\end{tabular}

Statistical significance $\mathrm{p}<0.05$ is marked in bold.

a diagnoses: 1 subject with bipolar I, most recent episode depressed; 2 subjects with bipolar I, mixed; 1 with bipolar I, manic; 1 with bipolar II; 5 with bipolar NOS; 4 with mood disorder NOS.

Table 4

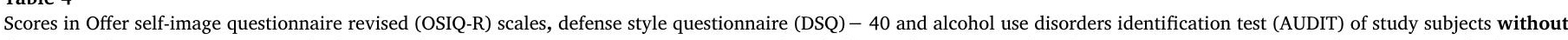
depression diagnosis: comparison of subjects with BDI-21 0-15 points or 16-63 points in the whole population (controls and patients), among patients and among control subjects.

\begin{tabular}{|c|c|c|c|c|c|c|c|c|c|}
\hline & \multicolumn{3}{|l|}{ ALL } & \multicolumn{3}{|l|}{ PATIENTS } & \multicolumn{3}{|l|}{ CONTROLS } \\
\hline & BDI $0-15 p$ & BDI $16-63 p$ & $\mathrm{p}\left(\mathrm{MW}^{*}\right)$ & BDI 0-15p & BDI $16-63 p$ & $\mathrm{p}\left(\mathrm{MW}^{*}\right)$ & BDI $0-15 p$ & BDI $16-63 \mathrm{p}$ & $\mathrm{p}\left(\mathrm{MW}^{\prime \prime}\right)$ \\
\hline \multicolumn{10}{|l|}{ mean (median; range) } \\
\hline OSIQ impulse control & $23(14-39 ; 226)$ & $34(21-49 ; 45)$ & 0.000 & $24(16-39 ; 39)$ & $34(21-49 ; 33)$ & 0.000 & $20(14-38 ; 187)$ & $36(23-39 ; 12)$ & 0.000 \\
\hline OSIQ emotional tone & $20(10-46 ; 225)$ & $42(20-57 ; 45)$ & 0.000 & $26(10-42 ; 38)$ & $47(27-59 ; 33)$ & 0.000 & $20(10-46 ; 187)$ & $38(25-51 ; 12)$ & 0.000 \\
\hline OSIQ mental health & $37(23-53 ; 224)$ & $47(27-59 ; 44)$ & 0.000 & $37(23-53 ; 38)$ & $47(27-59 ; 32)$ & 0.000 & $37(26-51 ; 186)$ & $46(37-51 ; 12)$ & 0.000 \\
\hline OSIQ social functioning & $20(9-36 ; 227)$ & $31(11-40 ; 43)$ & 0.000 & $24(10-34 ; 40)$ & $32(11-40 ; 31)$ & 0.000 & $19(9-36,187)$ & $28(16-40 ; 12)$ & 0.02 \\
\hline OSIQ family functioning & $40(20-90 ; 219)$ & $60(29-88 ; 43)$ & 0.000 & $41(22-90 ; 34)$ & $60(29-88 ; 31)$ & 0.005 & $39(20-88 ; 185)$ & $61(37-88 ; 12)$ & 0.000 \\
\hline OSIQ self confidence & $23(11-38 ; 228)$ & $34(19-48 ; 45)$ & 0.000 & $26(14-38 ; 41)$ & $34(19-48 ; 33)$ & 0.000 & $23(11-38 ; 187)$ & $34(27-39 ; 12)$ & 0.000 \\
\hline OSIQ self reliance & $39(24-63 ; 221)$ & $44(34-61 ; 45)$ & 0.000 & $42(27-63 ; 37)$ & $44(34-61 ; 33)$ & 0.08 & $39(24-62 ; 184)$ & $46(36-57 ; 12)$ & 0.007 \\
\hline OSIQ ethical values & $23(11-43 ; 224)$ & $27(15-45 ; 43)$ & 0.002 & $23(11-35 ; 38)$ & $26(15-45 ; 31)$ & 0.16 & $23(11-43 ; 186)$ & $30(20-41 ; 12)$ & 0.001 \\
\hline OSIQ body image & $20(8-38 ; 226)$ & $32(17-40,45)$ & 0.000 & $23(9-34 ; 39)$ & $33(18-40 ; 33)$ & 0.000 & $19(8-38 ; 187)$ & $30(17-35 ; 12)$ & 0.000 \\
\hline DSQ mature & $58(10-88 ; 228)$ & $46(18-71 ; 46)$ & 0.000 & $55(17-82 ; 44)$ & $46(18-71 ; 34)$ & 0.004 & $58(10-88 ; 184)$ & $47(20-54 ; 12)$ & 0.000 \\
\hline DSQ neurotic & $35(8-64 ; 230)$ & $39(16-60 ; 47)$ & 0.006 & $34(12 ; 44)$ & $42(16-60 ; 35)$ & 0.01 & $35(8-62 ; 186)$ & $35(30-46 ; 12)$ & 0.6 \\
\hline DSQ image-distorting & $34(10-83 ; 225)$ & $41(22-70 ; 45)$ & 0.001 & $34(12-64 ; 43)$ & $41(22-55 ; 33)$ & 0.1 & $34(10-63,182)$ & $40(31-70 ; 12)$ & 0.03 \\
\hline DSQ immature & $41(12-84 ; 227)$ & $64(37-90 ; 46)$ & 0.000 & $46(12-79 ; 43)$ & $65(39-90 ; 34)$ & 0.000 & $41(12-84 ; 184)$ & $65(37-81 ; 12)$ & 0.000 \\
\hline AUDIT sum & $0(0-25 ; 234)$ & $4(0-31 ; 46)$ & 0.001 & $0(0-25 ; 48)$ & $2(0-31 ; 34)$ & 0.01 & $1(0-20 ; 186)$ & $7(0-28 ; 12)$ & 0.000 \\
\hline
\end{tabular}

* MW Mann-Whitney $U$-test for independent samples. Statistical significance $\mathrm{p}<0.05$ is marked in bold.

over). The only statistically significant odds ratio was for OSIQ-R emotional tone scale (odds ratio 95\% confidence interval 1.1-1.4, $\mathrm{p}=0.003)$.

\subsubsection{Conclusion}

In conclusion, high BDI-21 scores in subjects without depression diagnosis were associated with female sex, late adolescence, more adverse life events and parents' psychosocial problems (comparatively in the control population in particular), more psychiatric diagnoses, worse self-image, more immature and less mature defense styles compared with subjects with BDI-21 scores under 16 and no depression diagnosis.

\subsection{Subjects with unipolar depression: comparisons based on BDI-21 dichotomous variable}

Among subjects with current DSM-IV unipolar depression diagnosis ( $n=105$ ), there were only 3 control subjects, and therefore we did not run the analyses separately with the control group. Of those with a diagnosis of depression, 23 subjects scored low in BDI-21 (sum score under 16) and 82 scored high in BDI-21.
3.3.1. Sex, age, adverse life events and parental psychosocial problems Background characteristics and most life stressors did not differ between those who scored low (under 16 points, $n=23$ ) and those who scored high in BDI-21 $(n=82)$. The only significant differences were that subjects with depression but low BDI-21 scores were more often ( $\mathrm{p}<0.006, \chi^{2}$ test) male $(9 / 23,39 \%)$ than those who scored higher in BDI-21 (males $11 / 82,13 \%$ ), and they had not been victim to sexual abuse ( $\mathrm{n}=0$ vs. $23 / 82,28 \%, \chi^{2}$ test $\mathrm{p}<0.005$ ).

\subsubsection{Psychiatric diagnoses}

Psychiatric diagnoses or their number did not differ significantly between groups (Table S2 in Supplementary material).

\subsubsection{Self-image and psychological defense mechanisms}

Subjects with unipolar depression but scoring low in BDI-21 had better self-image as measured with OSIQ-R compared with subjects with depression and scoring low in BDI-21 (Table 5).

In defense styles (measured with DSQ-40), no significant difference was observed in mature defenses, but neurotic, immature and image distorting defenses were less prevalent in subjects scoring low in BDI-21 
Table 5

Scores in Offer self-image questionnaire revised (OSIQ-R) scales, defense style questionnaire (DSQ) - 40 and alcohol use disorders identification test (AUDIT) of study subjects with unipolar depression diagnosis: comparison of subjects with BDI-21 0-15 points or 16-63 points in the whole population (controls and patients). Results for patients and control subjects are not shown separately because among control subjects, only 3 had current DSM-IV unipolar depression.

\begin{tabular}{llll}
\hline & \multicolumn{2}{l}{ ALL with unipolar depression } & \\
\cline { 2 - 4 } & BDI $0-15 \mathrm{p}$ & BDI $16-63 \mathrm{p}$ & $\mathrm{p}(\mathrm{MW}$ *) \\
\hline mean (st dev; n) & & & \\
age & $15.0(1.1 ; 23)$ & $15.2(1.2 ; 82)$ & 0.4 \\
OSIQ impulse control & $25(4 ; 22)$ & $33(6 ; 76)$ & $\mathbf{0 . 0 0 0}$ \\
OSIQ emotional tone & $28(7 ; 20)$ & $43(8 ; 75)$ & $\mathbf{0 . 0 0 0}$ \\
OSIQ mental health & $39(6 ; 22)$ & $48(7 ; 73)$ & $\mathbf{0 . 0 0 0}$ \\
OSIQ social functioning & $24(6 ; 22)$ & $30(8 ; 76)$ & $\mathbf{0 . 0 0 0}$ \\
OSIQ family functioning & $45(11 ; 18)$ & $62(16 ; 74)$ & $\mathbf{0 . 0 0 0}$ \\
OSIQ self confidence & $27(5 ; 22)$ & $36(7 ; 76)$ & $\mathbf{0 . 0 0 0}$ \\
OSIQ self reliance & $43(7 ; 22)$ & $48(8 ; 72)$ & $\mathbf{0 . 0 0 4}$ \\
OSIQ ethical values & $26(7 ; 19)$ & $27(7 ; 74)$ & 0.4 \\
OSIQ body image & $22(6 ; 22)$ & $32(6,75)$ & $\mathbf{0 . 0 0 0}$ \\
DSQ mature & $47(14 ; 21)$ & $42(13 ; 76)$ & 0.1 \\
DSQ neurotic & $31(11 ; 20)$ & $40(15 ; 78)$ & $\mathbf{0 , 0 1}$ \\
DSQ image-distorting & $30(11 ; 19)$ & $38(11 ; 75)$ & $\mathbf{0 , 0 1}$ \\
DSQ immature & $43(16 ; 20)$ & $59(15 ; 76)$ & $\mathbf{0 . 0 0 0}$ \\
AUDIT sum & $1.7(4 ; 20)$ & $5.0(7 ; 81)$ & $\mathbf{0 . 0 2}$ \\
\hline
\end{tabular}

and having depression compared with those scoring high in BDI-21 and having depression (Table 5).

\subsubsection{AUDIT}

Subjects with depression but BDI-21 under 16 scored lower in AUDIT than subjects with depression and high BDI-21 scores (Table 5).

\section{Discussion}

We investigated what psychological and background factors are associated with discrepancy between BDI-21 scores and unipolar depression diagnosis in an adolescent inpatient population and an ageand gender-matched sample from general population. The discrepancy between not fulfilling diagnostic criteria for unipolar depression but scoring high on BDI-21 was associated with female sex, negative selfimage and immature defense style as well as parents having psychiatric problems in the patient population where all subjects had psychiatric problems, often accompanied by social problems and adverse life events. Of adverse life events, being victim of sexual abuse was associated with high BDI-21 sum score in the absence of clinical depression in the patient population. In the general population, where neither psychiatric nor social problems were as common, high BDI-21 sum score without depression diagnosis was in addition associated with psychiatric morbidity, parental psychiatric problems and substance use as well as physical abuse at home and outside of home. Discrepancy between fulfilling diagnostic criteria for current depression but scoring low on BDI-21 was associated with better self-image, and neurotic, immature and image distorting defenses were less prevalent compared with subjects with depression and scoring high in BDI-21.

\subsection{BDI specificity and Comorbidity}

Our results exemplify the fact that the specificity of BDI-21 for depression may be adequate for community samples, but not necessarily for patient samples where comorbidity is high (Brooks 2004). In our control population, $6 \%$ (12 subjects) scored 16 or higher on BDI-21 in the absence of clinical depression, whereas in the patient population, the corresponding figure was $43 \%$. The high comorbidity in our patient population $(68 \%)$ is consistent with observations in adolescent clinical populations (Angold et al., 1999; Karlsson et al., 2006) and also in adolescents with subthreshold MDD (Carrellas et al., 2017).
Discrepancy between BDI scores and diagnostics may also reflect diagnostic uncertainties: discerning between depression and anxiety disorders is difficult even when applying K-SADS-PL, the gold standard for diagnostics in adolescents (Lauth et al., 2010), and different diagnostic strategies may lead to different outcomes (Youngstrom et al., 2003). On the other hand, high BDI sum scores may reflect dissatisfaction, anxiety and dysphoria rather than specific depressive symptoms (Brooks and Kutcher, 2001). In our study population, anxiety disorders do not, however, conclusively explain the discrepancy between high BDI-21 scores and absence of depression diagnosis. Among control subjects who manifested this discrepancy, substance use disorder was as prevalent as anxiety disorders (4/12 or 33\%). Substance use disorder often coincides with depression in adolescents (Kaminer et al., 2007), and has been associated with depressive symptoms measured with BDI-21 in adults (Moore et al., 2016). In our patient population among those with no depression diagnosis, anxiety disorder was not significantly more prevalent in those scoring high in BDI than in those scoring low. The observation that high BDI-21 scores even in the absence of depression were associated with female sex is in line with studies on adolescents that have reported that girls score on average as much as 10 points higher than boys in BDI-21 (Kumar et al., 2002) and that have reported subthreshold MDD to be more prevalent in girls than in boys (Carrellas et al., 2017).

\subsection{Familial psychiatric morbidity, adverse life events and sex}

We observed that parental psychiatric problems were significantly associated with the discrepancy of high BDI-21 scores and no clinical depression in both patient and control population. Parent's depression is an important risk factor for depression and other mental health problems in adolescence (Weissman et al., 2006; Rice et al., 2017). High BDI-21 scores in the absence of clinical depression may reflect clinical antecedent to MDD. Following this interpretation, our result does not, however, conform to a recent prospective study on offspring of depressed parents where clinical antecedent symptoms (irritability, fear/anxiety) were risk factors for MDD but they did not mediate the familial risk (Rice et al., 2017). Our study, like many previous studies, endorses the importance of preventive strategies aimed at parents with psychiatric problems and their children.

In regard to adverse life events, our most notable finding is that in patients without depression sexual abuse was more frequently reported among subjects who scored high in BDI-21 compared to those who scored low. The finding is in line with previous studies such as a recent longitudinal study on subjects from 4 years of age until 16 years that showed that sexual abuse in childhood was associated with higher prevalence of psychiatric symptoms as reported in Child Behavior Checklist by caregivers compared with other forms of maltreatment (Lewis et al., 2016). In the control population, adverse life events and problems in the family were reported only infrequently. Thus, the rather small number of cases prevents us from drawing any further conclusions regarding the general population.

Among subjects with depression, low BDI-21 scores were observed predominantly in males. The result suggests that psychiatric assessment of boys, in particular, should include depression diagnostics regardless of the BDI-21 score.

\subsection{Self-image}

Our findings that self-reported depressive symptoms are associated with negative self-image in most OSIQ scales is in line with previous studies on adolescents. OSIQ scales, especially Emotional Tone and Mastery of the External World (Self-Confidence) scales, have been reported to predict depression in adolescents (Fine et al., 1993). Significant correlation between self-reported depressive symptoms (high CDI scores) and negative self- image in all scales of OSIQ has been reported in a non-clinical adolescent population, where moreover, the 
correlation was stronger for females than males (Erkolahti et al., 2003). Regarding the association between clinical diagnosis and self-image our results, however, differ from some earlier findings. In a clinical population with varied diagnoses, the worst self-image as measured with OSIQ was observed in patients with MDD (Koenig, 1988), whereas dysphoria i.e. milder depressive symptoms than MDD, was not associated with any problems in self-image (Koenig, 1988). Furthermore, patients with recurrent depression had less problems in self-image than those suffering from first episode of MDD, although no significant difference in the severity of current episode was observed (Koenig, 1988). Koenig thus argued that OSIQ would seem to measure different psychological factors than depressive symptoms. In our study, however, subjects who did not have clinical depression but scored high in BDI-21 scored worse in OSIQ-R (had more problems with self-image) than those without depression and with low BDI-21 scores. Longitudinal studies are needed to better determine in what extent the association between negative self-image as measured with OSIQ and depressive symptoms represents a true association between clinical symptoms and psychological factors rather than the manifestation of depressive mood and thoughts in various self-reports.

\subsection{Defense styles}

Our study showed that adolescents who scored high on BDI-21 in absence of clinical depression relied more on immature defense styles and less on mature defense styles (as measured with DSQ-40) than those who scored low in BDI-21. This result on both non-clinical and clinical populations conforms with previous studies that have reported psychiatric symptoms to be associated with immature defense styles in non-clinical populations (Chan, 1997; Muris et al., 2003; Ruuttu et al., 2006) as well as clinical populations (Ruuttu et al., 2006). A metaanalysis on studies on clinical adult populations concluded that patients with MDD reported significantly lower scores in mature defense style and higher scores in neurotic and immature styles than control subjects (Calati et al., 2010). Muris et al. observed the association of depressive symptoms and immature defense styles among girls only, whereas in boys neurotic defense style was associated with depressive symptoms (Muris et al., 2003). Immature defense styles have been reported to also predict psychiatric symptoms in a longitudinal study on a non-clinical adolescent population (Tuulio-Henriksson et al., 1997). Also our observation that low BDI-21 scores in adolescents without depression were associated with mature defense styles is in agreement with previous reports on the association between mature defense styles and low prevalence of psychiatric symptoms (Muris et al., 2003; Ruuttu et al., 2006). In a five-year follow-up mature defense style was negatively associated with psychiatric problems, but only among female subjects (Tuulio-Henriksson et al., 1997). Muris et al. observed in their nonclinical study population that neurotic defense style was associated with various anxiety symptoms, among girls in particular (Muris et al., 2003).

\subsection{Strengths of the study}

The essential strength of our study is that we had a relatively large study population and both patients and age- and sex-matched control subjects. The study represents a naturalistic setting in inpatient care with high comorbidity and many confounding factors, which can be considered both strength and a weakness. Careful diagnostics was conducted in all subjects. The self-assessment questionnaires that we used have been validated for adolescents. Participation rate was relatively high, but unfortunately varied in different self-reports.

\subsection{Limitations of the study}

Our study population consists of two extremes: adolescents whose psychiatric problems require hospitalization and community-based sample of students recruited from their schools. The results may not be applicable to other kinds of populations such as adolescents seeking help in primary health care services. Interpretation of our results is limited by the fact that personality disorders (or traits of any) were not considered, which is due to the fact that assessing personality disorders in adolescents has been controversial. The psychological characteristics (defense styles, self-image) are based on structured self-reports only. Indepth psychological testing would, however, be resource intensive in such a large study population. Adverse life events in our study were recalled retrospectively and in the case of control subjects, only adolescents themselves were enquired. Information on parental mental health and substance use was also obtained from study subjects only in the control group. In the patient group, information was supplemented by patients' clinical records. Another methodological limitation is that we did not assess the inter-rater reliability of K-SADS-PL interviews. On the other hand, all diagnoses were confirmed in diagnostic consensus meeting with experienced adolescent psychiatrists.

\subsection{Conclusions}

Our study showed that adolescents who report many depression symptoms in BDI-21 but do not fulfill diagnostic criteria for current depression share many characteristics that in previous studies have been associated with depression and depressive symptoms. Even if she/ he does not fulfill the criteria for depression, high scores in self-report BDI-21 merit a broader assessment of an adolescent's psychological and social wellbeing and need for support. Addressing adolescent's selfimage and defense styles may be warranted, and on the other hand parents' psychiatric problems may be one key intervention.

\section{Acknowledgements}

We are grateful to Erkki Pulkkinen, MD $\mathrm{PhD}$, for his intellectual input and enduring support. We also would like to warmly thank all the medical doctors specializing in adolescent psychiatry as well as all the personnel at the Kellokoski adolescent psychiatric ward who took care of the patients and were involved in gathering data for this study.

\section{Funding}

This work was funded by the Helsinki University Hospital Research funding and Hyvinkää Hospital District.

\section{Appendix A. Supplementary material}

Supplementary data associated with this article can be found in the online version at http://dx.doi.org/10.1016/j.psychres.2018.02.040.

\section{References}

Ambrosini, P.J., 2000. Historical development and present status of the schedule for affective disorders and schizophrenia for school-age children (K-SADS). J. Am. Acad. Child Adolesc. Psychiatry 39, 49-58.

Angold, A., Costello, E.J., Erkanli, A., 1999. Comorbidity. J. Child Psychol. Psychiatry 40, 57-87.

Avenevoli, S., Swendsen, J., He, J.P., Burstein, M., Merikangas, K.R., 2015. Major depression in the national comorbidity survey-adolescent supplement: prevalence, correlates, and treatment. J. Am. Acad. Child Adolesc. Psychiatry 54, 37-44 (e2).

Beck, A.T., Ward, C.H., Mendelson, M., Mock, J., Erbaugh, J., 1961. An inventory for measuring depression. Arch. Gen. Psychiatry 4, 561-571.

Brooks, S.J., Kutcher, S., 2001. Diagnosis and measurement of adolescent depression: a review of commonly utilized instruments. J. Child Adolesc. Psychopharmacol. 11, $341-376$.

Calati, R., Oasi, O., De Ronchi, D., Serretti, A., 2010. The use of the defence style questionnaire in major depressive and panic disorders: a comprehensive meta-analysis. Psychol. Psychother. 83, 1-13.

Carrellas, N.W., Biederman, J., Uchida, M., 2017. How prevalent and morbid are subthreshold manifestations of major depression in adolescents? A literature review. J. Affect. Disord. 210, 166-173.

Chan, D.W., 1997. Defensive styles and psychological symptoms among Chinese 
adolescents in Hong Kong. Soc. Psychiatry Psychiatr. Epidemiol. 32, 269-276.

(St)Clair, M.C., Croudace, T., Dunn, V.J., Jones, P.B., Herbert, J., Goodyer, L.M., 2015. Childhood adversity subtypes and depressive symptoms in early and late adolescence. Dev. Psychopathol. 27, 885-899.

Dolle, K., Schulte-Korne, G., O'Leary, A.M., von Hofacker, N., Izat, Y., Allgaier, A.K., 2012. The Beck depression inventory-II in adolescent mental health patients: cut-off scores for detecting depression and rating severity. Psychiatry Res. 200, 843-848.

Erkolahti, R., Ilonen, T., Saarijarvi, S., Terho, P., 2003. Self-image and depressive symptoms among adolescents in a non-clinical sample. Nord. J. Psychiatry 57, 447-451.

Fine, S., Haley, G., Gilbert, M., Forth, A., 1993. Self-image as a predictor of outcome in adolescent major depressive disorder. J. Child Psychol. Psychiatry 34, 1399-1407.

Kaminer, Y., Connor, D.F., Curry, J.F., 2007. Comorbid adolescent substance use and major depressive disorders: a review. Psychiatry (Edgmont) 4, 32-43.

Karlsson, L., Pelkonen, M., Ruuttu, T., Kiviruusu, O., Heila, H., Holi, M., Kettunen, K., Tuisku, V., Tuulio-Henriksson, A., Torronen, J., Marttunen, M., 2006. Current comorbidity among consecutive adolescent psychiatric outpatients with DSM-IV mood disorders. Eur. Child Adolesc. Psychiatry 15, 220-231.

Kaufman, J., Birmaher, B., Brent, D., Rao, U., Flynn, C., Moreci, P., Williamson, D., Ryan, N., 1997. Schedule for affective disorders and schizophrenia for school-age childrenpresent and lifetime version (K-SADS-PL): initial reliability and validity data. J. Am. Acad. Child Adolesc. Psychiatry 36, 980-988.

Knight, J.R., Sherritt, L., Harris, S.K., Gates, E.C., Chang, G., 2003. Validity of brief alcohol screening tests among adolescents: a comparison of the AUDIT, POSIT, CAGE, and CRAFFT. Alcohol. Clin. Exp. Res. 27, 67-73.

Koenig, L.J., 1988. Self-image of emotionally disturbed adolescents. J. Abnorm. Child Psychol. 16, 111-126.

Kumar, G., Steer, R.A., Teitelman, K.B., Villacis, L., 2002. Effectiveness of Beck Depression Inventory-II subscales in screening for major depressive disorders in adolescent psychiatric inpatients. Assessment 9, 164-170.

Laukkanen, E., Halonen, P., Aivio, A., Viinamäki, H., Lehtonen, J., 2009. Construct validity of the Offer self-image questionnaire in Finnish 13-year-old adolescents: differences in the self-images of boys and girls. Nord. J. Psychiatry 54, 431-435.

Lauth, B., Arnkelsson, G.B., Magnusson, P., Skarpheethinsson, G.A., Ferrari, P., Petursson, H., 2010. Validity of K-SADS-PL (schedule for affective disorders and schizophrenia for school-age children-present and lifetime version) depression diagnoses in an adolescent clinical population. Nord. J. Psychiatry 64, 409-420.

Lewis, T., McElroy, E., Harlaar, N., Runyan, D., 2016. Does the impact of child sexual abuse differ from maltreated but non-sexually abused children? A prospective examination of the impact of child sexual abuse on internalizing and externalizing behavior problems. Child Abus. Negl. 51, 31-40.

Mason, W.A., Chmelka, M.B., Trudeau, L., Spoth, R.L., 2017. Gender moderation of the intergenerational transmission and stability of depressive symptoms from early adolescence to early adulthood. J. Youth Adolesc. 46, 248-260.

Moore, A.A., Neale, M.C., Silberg, J.L., Verhulst, B., 2016. Substance use and depression symptomatology: measurement invariance of the Beck Depression Inventory (BDI-II) among non-users and frequent-users of alcohol nicotine and cannabis. PLOS One 11, e0152118.

Muris, P., Winands, D., Horselenberg, R., 2003. Defense styles personality traits and psychopathological symptoms in nonclinical adolescents. J. Nerv. Ment. Dis. 191, $771-780$.

Mustanoja, S., Luukkonen, A.H., Hakko, H., Rasanen, P., Saavala, H., Riala, K., STUDY-70 workgroup, 2011. Is exposure to domestic violence and violent crime associated with bullying behaviour among underage adolescent psychiatric inpatients? Child Psychiatry Hum. Dev. 42, 495-506.

Offer, D., Ostrov, J.D., Howard, K.I., Dolan, M.A., 1992. Offer Self-image Questionnaire Revised (OSIQ-R) Manual. Western Psychological Services. Los Angeles, California.

Osman, A., Barrios, F.X., Gutierrez, P.M., Williams, J.E., Bailey, J., 2008. Psychometric properties of the Beck Depression Inventory-II in nonclinical adolescent samples. J. Clin. Psychol. 64, 83-102.

Rice, F., Sellers, R., Hammerton, G., Eyre, O., Bevan-Jones, R., Thapar, A.K., Collishaw, S., Harold, G.T., Thapar, A., 2017. Antecedents of new-onset major depressive disorder in children and adolescents at high familial risk. JAMA Psychiatry 74, 153-160.

Ruuttu, T., Pelkonen, M., Holi, M., Karlsson, L., Kiviruusu, O., Heila, H., Tuisku, V., Tuulio-Henriksson, A., Marttunen, M., 2006. Psychometric properties of the defense style questionnaire (DSQ-40) in adolescents. J. Nerv. Ment. Dis. 194, 98-105.

Rytila-Manninen, M., Lindberg, N., Haravuori, H., Kettunen, K., Marttunen, M. Joukamaa, M., Frojd, S., 2014. Adverse childhood experiences as risk factors for serious mental disorders and inpatient hospitalization among adolescents. Child Abus. Negl. 38, 2021-2032.

Stockings, E., Degenhardt, L., Lee, Y.Y., Mihalopoulos, C., Liu, A., Hobbs, M., Patton, G., 2015. Symptom screening scales for detecting major depressive disorder in children and adolescents: a systematic review and meta-analysis of reliability, validity and diagnostic utility. J. Affect. Disord. 174, 447-463.

Thapar, A., Collishaw, S., Pine, D.S., Thapar, A.K., 2012. Depression in adolescence. Lancet 379, 1056-1067.

Tuisku, V., Pelkonen, M., Karlsson, L., Kiviruusu, O., Holi, M., Ruuttu, T., Punamaki, R.L., Marttunen, M., 2006. Suicidal ideation, deliberate self-harm behaviour and suicide attempts among adolescent outpatients with depressive mood disorders and comorbid axis I disorders. Eur. Child Adolesc. Psychiatry 15, 199-206.

Tuulio-Henriksson, A., Poikolainen, K., Aalto-Setala, T., Lonnqvist, J., 1997. Psychological defense styles in late adolescence and young adulthood: a follow-up study. J. Am. Acad. Child Adolesc. Psychiatry 36, 1148-1153.

Weissman, M.M., Wickramaratne, P., Nomura, Y., Warner, V., Pilowsky, D., Verdeli, H., 2006. Offspring of depressed parents: 20 years later. Am. J. Psychiatry 163 , 1001-1008.

Youngstrom, E.A., Findling, R.L., Calabrese, J.R., 2003. Who are the comorbid adolescents? Agreement between psychiatric diagnosis, youth, parent, and teacher report. J. Abnorm. Child Psychol. 31, 231-245.

Youngstrom, E.A., Youngstrom, J.K., Freeman, A.J., De Los Reyes, A., Feeny, N.C., Findling, R.L., 2011. Informants are not all equal: predictors and correlates of clinician judgments about caregiver and youth credibility. J. Child Adolesc. Psychopharmacol. 21, 407-415. 